\title{
Beiträge zur Lehre von den Aderhantsarkomen.
}

Von

\author{
Dr. Otto Bruns, \\ Assistenten an der Universitäts-Augenklinik zu Heidelberg. \\ Mit Taf. XII, Fig. 1-3 and 4 Figuren im Text.
}

Wenn ich die schon überreiche Casuistik der Aderhautsarkome um zwei weitere Fälle vermehre, so glaube ich es durch das in mehrfacher Hinsicht ungewöhnliche histologische Verhalten derselben rechtfertigen zu können.

Ich lasse nachstehend die Krankengeschichten und anatomischen Befunde folgen.

$$
\text { Fall 1. }
$$

Engelbert V. aus G., Schuhmacher, 51 Jahre.

Der bis dahin stets gesunde Mann will angeblich erst seit einigen Wochen vor dem linken Auge einen Schatten bemerken, der das Sehen empfindlich stört.

Klinischer Befund: Rechtes Auge. Aensserlich und ophthalmoskopisch vollkommen normal. E. S. $=1$.

Linkes Auge erscheint äusserlich ebenfalls durchaus normal. In der Gegend- des hinteren Augenpoles findet sich eine halbkugelförmige kreisrunde, ganz scharf begrenzte Netzhautablösung, die sich durch ihre grauweisse Farbe vom übrigen Augenhintergrunde sehr deutlich abhebt. Dieselbe ist im horizontalen Meridian derart gelegen, dass der Abstand ihres nasalen Randes vom Selınerveneintritt nicht ganz zwei Papillenbreiten beträgt. Ihr Durchmesser übersteigt zwei Papillenbreiten um weniges. Diese stark prominirende Netzhautpartie, die eine Refractionsdifferenz von 8 bis $10 \mathrm{D}$ aufweist, ist ringförmig umgeben von einer seichten, sich allmählich in den normalen Augenhintergrund verlierende Abhebung von graurother Farbe. Ihr Durchmesser schwankt zwischen einer und zwei Papillenbreiten und ist auf der temporalen Seite grösser als auf der nasalen. Die Netzhautgefässe lassen sich überall mit vollkommener Deutlichkeit und ohne Unterbrechungen verfolgen. Während die kugelige Prominenz von der Netzhaut glatt und faltenlos überzogen wird, lässt die seichte ringförmige Abhebung zahlreiche feine, radiär verlaufende Netzhauttältchen erkennen.

Der Sehnerveneintritt sowie der übrige Augenhintergrund weisen keine Besonderheiten auf. Der Tonus ist normal. Der Netzhautablösung entspricht 
ein centrales Skotom mit excentrisch nach dem temporalen Rand gelegenem Fixirpunkt.

Auf Grund dieses Befundes warde die Diagnose auf Aderhauttumor gestellt und das Auge am 22. August 1901 enucleirt. In der Orbitalhöhle fand sich keine Geschwulstmetastase; die Sklera des hinteren Bulbusabschnittes und der Opticusquerschnitt sehienen intact zu sein. Der Patient wurde am 2. September nach normalem Heilungsverlauf aus der Behandlung entlassen. Ueber sein weiteres Schicksal ist mir nichts bekannt.

Der Bulbus wurde in $10 \%$ Formalinlösung fixirt, in aufsteigendem Alkohol gehärtet, in Celloidin eingebettet und in Serienschnitte zerlegt.

\section{Anatomische Untersuchung.}

A. Makroskopischer Befund: Die Grössenverhältnisse des Bulbus sind normal. Der äquatoriale Durchmesser beträgt $24 \mathrm{~mm}$, der anteroposteriore $25 \mathrm{~mm}$. Der vordere Bulbusabschnitt zeigt nichts Bemerkenswerthes. Die vordere Kammer ist von normaler Tiefe, der Kammerwinkel ist frei.

In der Gegend des hinteren Augenpoles, etwas temporal vom Sehnerveneintritt erhebt sich ein kirschkerngrosser Tumor, der die bekannte, der Durchbruchsstelle durch die Glaslamelle entsprechende gürtelförmige Einschnïrung in sehr ausgesprochener Weise zeigt. Die Basis, d. h. der intrachorioideale Theil hat einen Durchmesser von $6 \mathrm{~mm}$, eine Höhe von $2 \mathrm{~mm}$. Der Durchmesser des die Lücke der Glaslamelle ausfüllenden Halses beträgt $2 \mathrm{~mm}$, der - subretinale - Kopf hat die Gestalt einer abgeplatteten Kugel, eine Höhe von $3 \mathrm{~mm}$ und eine Breite ron $4 \mathrm{~mm}$.

Die Netzhaut liegt der ganzen Kuppe des Tumors fest an. In seiner Umgebung besteht eine ziemlich ausgedehnte, nasal bis zum Sehnerveneintritt, temporalwärts bis zum Aequator sich erstreekende Netzhautablösung, die durch ein eiweissreiches, unter dem Einfluss der Härtungsmittel geromnenes subretinales Exsudat bedingt ist und deren Höhe mit der Entfernung rom Tumor continuirlich abnimmt. An einigen Stellen, namentich im oberen Abschnitt des Fundus, ist die Retina ausserdem noch von dieser Exsudatschicht blasenförmig abgehoben, fraglos ein Artefact.

Auf dem Durchschnitt ist der Tumor von weisser Farbe, nur die Basis ist in ihren Randpartien leicht pigmentirt.

B. Mikroskopischer Befund: Die Geschwulst setzt sich zusammen aus typischen Spindelzellen, die dicht gedrängt in vielfach sich kreuzenden Bündeln angeordnet sind. Das sehr spärliche Stroma lässt sich nur an den dünnsten Stellen der Präparate als feinstes Fasernetz nachweisen. Die Zellkerne sind gross, bläschenförmig mit einem, bisweilen zwei sehr deutlichen Kernkörperchen. Karyokinetische Figuren sind in nicht erheblicher Zahl in allen Theilen der Gesehwulst zu finden.

Auffallend ist der grosse Gefässreichthum besonders des extrachorioidealen Antheils. An manchen Stellen wird man geradezu an das Bild des Angiosarkoms gemahnt, doch fehlt die für dasselbe charakteristische radiäre Anordnung der Geschwulstzellen um die Gefässe herum vollständig.

Zum überwiegend grössten Theil ist die Neubildung pigmentfrei. Nur auf der Kuppe, dort wo sie mit der Retina in Contact steht, sind spärliche 
Pigmentzellen anzutreffen, die dureh ihre fächenhafte Anordnung und ihre kugelige oder polyedrisehe Gestalt ihre Provenienz aus dem Pigmentepithel unzweifelhaft darthun und von dem wachsenden Tumor hierher transportirt worden sind. Entsprechend liegen zwischen den Zellen der Geschwulstbasis zerstreut verzweigte, pigmentführende Zellen, die als Reste des im Tumor aufgegangenen Stromapigmentes aufzufassen sind. Während der grösste Theil der Gesehwulst in der beschriebenen Weise das typisehe Bild des Lenkosarkoms darbietet, lässt sieh durch Seriensehnitte nachweisen, dass in der Achse des Tumors ein aus Pigmentzellen bestehender Strang verläuft, der von den Stromapigmentresten der Basis ausgehend den Tumorhals durchzieht, um sich noch eine Strecke weit im subretinalen Geschwulstknoten zu verbreiten. Diese Pigmentzellen sind langgestreckt, verzweigt und zeigen mit den Blutgefässen gleiche Verlaufsrichtung. $\mathrm{Zu}$ den Geschwulstzellen stehen sie in keiner Beziehung, vielmehr sind diese stets pigmentfrei.

Ein Eisengehalt des Geschwulstpigmentes sowohl, wie des Pigmentepithels war mit Hilfe der Perls'schen Reaction trotz wiederholter Versuche nicht nachzuweisen.

Die Netzhaut zeigt eine durch ein homogenes, sich mit Eosin stark färbendes Exsudat bedingte seichte, aber ziemlich ausgedehnte Ablösung. Sie erstreckt sich vom Sehnerveneintritt aus temporalwärts und nach unten bis zum Aequator, während sie nach oben in kurzer Entfernung von der Geschwulst ihr Ende findet. Nur in unmittelbarem Bereich des Sarkoms erlangt die Abhebung eine erhebliche Höhe, indem die Retina hier mit der Kuppe des Tumors in grosser Ausdehnung Verwachsungen eingegangen ist. Das Verhalten der einzelnen Retinaschichten ist nun so eigenartig, dass es eine nähere Betrachtung verdient.

Wenn ich vom Pigmentepithel absehe, welches ich trotz seiner Zugehörigkeit zur Retina erst später besprechen will, so ist es zunächst die äussere Körnersehicht, welche die Aufmerksamkeit auf sich lenkt. Kurz bevor sie mit dem Tamor in Berührung tritt, beginnt sie in eigenthümlicher Weise sich zu verbreitern, nicht durch Zellvermehrung, sondern durch Auflockerung des Gewebes. So kommt es schliesslich zu einer mehr oder weniger deutlichen Spaltung in zwei Zellschichten, die durch ein weitmasehiges, aus den Stützfasern bestehendes Fasernetz zusammengehalten werden. Sobald die Retina den Geschwulstrand erreicht hat, biegt die äussere dieser beiden Zellschichten in scharfem Winkel in der Richtung auf die Tumorbasis um, die Geschwulst kapselartig umhüllend. Dabei findet, je mehr sie sich der Einschnürungsstelle nähert, eine Verdünnung statt. Die Zellen verlieren ihren Rundzellencharakter und wandeln sich in platte, Bindegewebszellen gleichende Elemente $u m$, bis sie schliesslich in Gestalt einer äusserst feinen Membran an der Durchbruchsstelle der Lamina interna ihr Ende findet, stellenweise auch mit dieser verwächst. Die innere Lamelle der änsseren Körnerschicht lässt sich noch eine Strecke weit auf der Tumorkuppe verfolgen, wird aber bald von Geschwulstmassen vielfach durchbrochen und schwindet schliesslich ganz.

Die soeben gegebene Beschreibung ist vor Allem für den unteren Rand des Tumors zutreffend. Für den oberen Rand bedarf sie einer 
Modification. Hier findet keine Spaltung der äusseren Körnerschicht statt, sondern sie trennt sich vöilig von der Hauptmasse der Retina und zieht, den Tumor bekleidend, direct zum Geschwulsthalse. Dagegen gilt das oben Gesagte hier in allen Stücken für die innere Körnerschicht, nämlich die Spaltung in zwei Blätter, deren äusseres gemeinsam mit der äusseren Kömersehicht nach der Basis des Sarkoms zustrebt und mit der Glaslamelle verwächst, während das innere Blatt mit geringen Unterbrechungen den Geschwulstrücken überzieht. Das beigefügte Mikrophotogramm (Taf. XIII, Fig. 1) lässt alle diese Verhältnisse mit genügender Deutlichkeit erkennen.

Die beiden Körnerschichten gemeinsam stellen somit eine fast allseitig geschlossene, den extrachorioidealen Antheil des Sarkoms umschliessende Kapsel dar.

Die übrigen Retinaschichten sind weniger von Interesse. Die Ganglienzellenschicht ist im Bereich der Verwachsung mit dem Tumor stark gewuchert. Ihre Elemente zeigen nicht mehr Form und Grösse der Ganglienzellen, sondern haben das Aussehen von Rundzellen angenommen. Die Nervenfaserschicht dagegen, die hier in der Nähe des Sehnerveneintritts eine bedeutende Mächtigkeit haben sollte, ist erheblich verdiunnt, stellenweise bis auf kaum sichtbare Reste.

Die Schilderung der oben mitgetheilten Verhältnisse bedarf insofern einer Ergänzung, als es der Proliferationskraft der Neubildung gelungen ist, an umschriebenen Stellen die sie umschliessende Kapsel zu sprengen. Namentlich am Aequator des Geschwulstkopfes hat eine ziemlich ausgedehnte Perforation der hier freilich nur aus einem Theil der Retinaschichten gebildeten Kapsel stattgefunden, und die Tumorzellen wuchern hier, pilaförmige Excrescenzen bildend, frei in das subretinale Exsudat hinein. Dieser Umstand ist aus Gründen, die weiter unten erörtert werden, von besonderer Wichtigkeit. Einen wirksameren Widerstand vermochte die Retina auf dem Geschwulstrücken zu leisten. Man sieht hier auf Serienschnitten das Eindringen der Tumorzellen zwischen die Zellagen der anliegenden Netzhautschichten, daselbst zu kleinen Knötchen auswachsend, so dass in manchen Schnitten vom Tumor scheinbar isolirte Geschwulstherde innerhalb der Retina sich vorfinden. Weiterhin kommt es dann zu einem völligen Durchbruch der Körmersehichten und die Neubildung berüht unmittelbar die an dieser Stelle stark verdünnte Ganglienzellenschicht. Nirgends indessen ist hier eine vollständige Perforation der Netzhaut naehzuweisen. Nirgends also tritt der Tumor in directe Berührung mit dem Glaskörper.

Die Beziehungen des Sarkoms zu seinem Mutterboden, zur Chorioidea sind die üblichen. Diese beginnt schon in beträchtlicher Entfernung breiter zu werden, theils durch ödematöse Auflockerung des Gewebes, besonders aber durch hochgradige Erweiterung der Gefässe der Haller'schen Schicht. Innerhalb der letzteren beginnt die Geschwulst ziemlich unvermittelt, nur in ibrer nächsten Nähe ist das Aderhantstroma von einzelnen Spindelzellen infiltrirt. Die Choriocapillaris lässt sich noch eine Strecke weit anf dem Neoplasma verfolgen, kann also nicht als Matrix desselben angesprochen werden.

Besonderes Interesse beansprucht das Pigmentepithel, welches ich wegen seiner räumlichen Zugehörigkeit - es ist wie fast stets bei der Netzhaut- 
ablösung an der Glaslamelle haften geblieben - an dieser Stelle erörtern möchte. In den vordersten Bulbusabsehnitten noch durchaus normal, beginnt es schon in weiter Entfernung vom Tumor, etwa in der Aequatorialgegend sein regelmässiges Aussehen zu verlieren. Durch ungleichartige Grösse der einzelnen Zellen entstehen Niveaudifferenzen, manche Zellen haben sich ganz aus dem Zusammenhang gelöst und liegen frei im subretinalen Raum. An anderen Stellen ist das Pigmentepithel in lebhafter Wucherung; woraus kugelige oder flache, ins Augeninnere vorspringende Prominenzen resultiren. Endlich aber finden sich in beschränkter Anzahl geschwulstartige Ansammlungen nicht pigmentirter Zellen, welche der Glaslamelle unmittelbar aufsitzen und das Pigmentepithel abgehoben haben, welehes sie als einschichtige Kapsel meist umhüllt, bisweilen aber eine Oeffnung zeigt, dureh welche die wuchernden Zellen in den subretinalen Raum gleichsam hervorquellen (Taf. XIII, Fig, 2). Die Kerne dieser Zellen sind meist etwas grösser als die des Pigmentepithels, rund oder leicht oval, bläschenartig mit sehr deutlichen Kernkörperchen. Weder zu diesen Gebilden, noch zu den vorher genannten Pigmentwucherungen zeigt die Glaslamelle irgend welehe Beziehungen. Sie lässt sich mit stets gleichbleibender Dicke bis zu ihrer Druchbruchsstelle verfolgen. Hier ist der freie Rand in der Wachsthumsichtung des Sarkoms nach dem Augeninneren zu spiralig eingerollt. Denkt man sich diese Spirale wieder aus einander gerollt, so bleibt nur ein sehr kleiner thatsächlicher Defect der Glaslamelle übrig. Nimmt man endlich eine auch nur geringe elastische Contractionsfähigkeit der Lamina interna an, so ist man zu dem Schluss berechtigt, dass keine Usur, nur eine Zerreissung derselben stattgefunden hat.

Ungeachtet des Frähstadiums, in dem die Neubildung sich noch befindet, ist bereits ein Uebergriff auf das Skleralgewebe eingeleitet, und $z$ war wie gewöhnlich in der Weise, dass kleine an dieser Stelle das Augeninnere verlassende Emissarien - in Betracht kommen vorzugsweise die Arteriae ciliares breves - als Austrittspforte benutzt wurden. Es lässt sich sehr schön verfolgen, wie in den Lymphscheiden dieser kleinen Gefässe die Geschwulstzellen fortgewandert sind und von hieraus die Lymphspalten der Sklera infiltrirt haben. Immerhin jedoch ist diese Propagation noch auf die ersten Anfänge beschränkt und nur auf wenigen Schnitten sichtbar, und nichts rechtfertigt den Verdacht, dass schon das retrobulbäre Gewebe in Mitleidenschaft gezogen sein könnte.

Der Sehnerveneintritt zeigt normale Verhältnisse. Seine Excavation überschreitet nicht das physiologische Mass. In den Sehnervenscheiden sind keine Geschwulstzellen anzutreffen. Ebenso erweist sich der vordere Bulbusabschnitt als in jeder Hinsicht intact.

\section{Fall 2.}

Babette J., 39 Jahre, Gymnasiallehrersfrau aus F.

Bemerkt seit zwei Monaten einen allmählich an Grösse zunehmenden Schatten vor dem linken Ange, war früher niemals angenleidend.

Klinischer Befund: Rechtes Auge in jeder Hinsicht normal. E. S. $=1$. Linkes Auge zeigt äusserlich keinerlei Anzeichen eines Krankheitsprocesses. Ophthalmoskopisch bemerkt man im ganzen oberen und äusseren Quadranten 
eine vollständig kugelig vorspringende, kreisrunde, blasenförmige Netzhautablösung. Der untere und der der Papille zugekehrte Rand ist in seinem ganzen Verlauf zu verfolgen, nach oben und aussen ist die Grenze mit dem Augenspiegel nicht mehr zu erreichen. Die Oberfläche der Blase hat weissröthliche Farbe, die Netzhautgefässe sind, nur auf kurze Strecken unterbrochen, sichtbar; an einzelnen Stellen finden sich isolirte kleine Haemorrhagien. Der übrige Angengrund ist normal, auch die Papille zeigt die gewöbnliche Färbung und physiologische Excavation. Der Glaskörper ist frei von Trübungen.

Der Vorwölbung entspricht eine halbkreisförmige, den Fixirpunkt nicht ganz erreichende Gesichtsfeldeinschränkung im nasalen unteren Quadranten. E. $S .=5 / 10$.

Ein Versuch, die abgehobene Stelle von aussen her mit dem Glühlämpchen zu durchlenchten, ergiebt, dass man es mit einer undurchsichtigen Blase zu thun hat. Nur der Rand ist etwas durchscheinend, das Centrum des Buckels lässt sich nicht durchleuchten.

Es wird danach die Diagnose auf Tumor gestellt, und das Auge am 28. Mai 1901 enucleirt. Die Bulbuskapsel schien intact, ein retrobulbärer Tumor war nicht vorhanden.

\section{Anatomische Untersuchung.}

Die Technik war dieselbe wie in Fall 1: Formalinfixirung, Alkoholhärtung, Celloidinserienschnitte.

A. Makroskopischer Befund. Der gehärtete Bulbus hat einen äquatorialen Durchmesser von $23 \mathrm{~mm}$, einen antero-posterioren von $21 \mathrm{~mm}$. Diese Abplattung von vorn nach hinten findet ihre Erklärung in einer flachen Delle am hinteren Pol, die am frisehen Bulbus nicht vorhanden war und erst unter dem Einfluss der Fixirungsmittel entstanden ist.

Im oberen äusseren Quadranten, ziemlich genau im Aequator, sitzt ein pilzförmiger Tumor von der Grösse einer dicken Erbse der Sklera breitbasig auf. Eine tiefgehende Einschnürung theilt ihn in zwei ungleiche Abschnitte. Die kleinere, innerhalb der Chorioidea gelegene Basis ist von kegelförmiger Gestalt, $10 \mathrm{~mm}$ breit, $4 \mathrm{~mm}$ hoch; der grössere, subretinale Kopf ist kngelig, $10 \mathrm{~mm}$ breit, $6 \mathrm{~mm}$ hoch. Der Geschwulsthals zeigt einen grössten Durchmesser von $5 \mathrm{~mm}$. Auf dem Durehschnitt ist das Neoplasma von weissröthlicher Farbe und lockerem Gefüge.

Die Netzhaut ist durch die Gesehwulst abgehoben und, so weit noch vorhanden, mit dem ganzen Rücken des extrachorioidealen Knotens fest verwachsen. Erst an der Stelle seiner grössten Circumferenz trennt sie sich von ihm und zieht, die Einschnürung überbrückend, in leichten Bogen zur Aderhaut. Es besteht somit zwischen ihr und der Basis der Neubildung ein dieselbe ringförmig umgebender, auf dem Durchschnitt rhombischer Canal, der dureh ein eiweissreiches, geronnenes Exsudat ausgefült ist. Die Netzhaut des übrigen Augengrundes zeigt nur arteficielle Abhebungen, die sich von der pathologisehen leicht durch das fehlende subretinale Exsudat unterscheiden lassen.

Ausser einer mässigen Verdünnung des mit dem Tumor in Berührung stehenden Skleraabschnittes ist an den übrigen Augentheilen nichts Besonderes wahrzunehmen. 
B. Mikroskopiseher Befund. Die Neubildung ist ein Sarkom, aufgebaut aus spindelzelligen Elementen mit grossen bläschenförmigen Kernen und deutlichen Kernkörperchen. Mitosen finden sich nur in spärlicher Zahl. An dünnen Stellen der Präparate ist leicht ein ziemlich reichlich entwickeltes, feinfädiges Stroma zu unterseheiden, in dem die Geschwulstzellen in lockeren Complexen eingebettet sind. Der Tumor ist ansserordentlich stark vaseularisirt. Die Gefässe sind in allen Grössen vorhanden, von $1 \mathrm{~mm}$ Durchmesser bis zu feinsten Capillaren. Um die Gefässe herum haben sich die Sarkomzellen in regelmässigen radiären Reihen angeordnet, so dass die Gefässquerschnitte von Rosetten, die Längsschnitte von Zellpallisaden umgeben scheinen.

Unabhängig von diesem Gefässnetz ist das Neoplasma noch von einem System mit einander communieirender Spalträume von zum Theil recht beträ̈chtlicher Weite durehsetzt. An vielen Stellen lässt sich eine deutliche Endothelauskleidung erkennen, an anderen scheint eine Wandung gänzlich zu fehlen, so dass die Geschwulstzellenstränge selbst die mehr oder weniger geradlinige Begrenzung abgeben. Während die Blutgefässe stets mit wohl erhaltenen, wenn auch mit Easin seblecht tingibeln rothen Blutkörperchen vollgestopft sind, enthalten diese Gewebsspalten spärliche polynucleäre Lenkocyten, stellenweise in Gruppen angehäuft, ferner körnigen Detritus, grosse kernlose, keinerlei Färbung annehmende glasige Schollen und braunrothe, wohl aus Blutfarbstoffderivaten bestehende Krystalldrusen.

Eine directe Communication dieser beiden Arten von Hohlräumen ist nirgends nachweisbar, dagegen lässt sich an zahlreichen Stellen beobachten, dass Blutgefässe innerhalb jener Spalträume und parallel mit ihnen verlaufen, von ihnen also scheidenartig eingeschlossen werden.

Die Gesehwulst ist ein wahres Lenkosarkom; die wenigen Pigmentzellen, die überhaupt vorhanden sind, documentiren sich durch Lage und Gestalt unzweifelhaft als Reste des zu Grunde gegangenen Stroma- bezw. Netzhautpigmentes. Mehrfach angestellte Eisenreactionen ergaben negative Resultate.

Die Retina, die, wie erwähnt, im unmittelbaren Umkreis der Geschwulst auf eine kaxze Strecke hin abgehoben ist, zeigt sehon in einiger Entfernung eine Auffaserung ihrer Sehichten. Insbesondere die Körnersehichten lockern und verbreitern sich auf Kosten der änsseren plexiformen Schicht, bis diese eine nur noch undeutliche Abgrenzung der Körnersebichten darstellt. Hand in Hand damit geht eine Hypertrophie der Müller'sehen Stützfasern, die als grossmaschiges Netzwerk immer mehr zur Geltung kommen. Bei Berührung mit dem. Tumorrand geht in einer mit Fall 1 analogen Weise eine Spaltung der äusseren Körnerschicht in zwei Blätter vor sich, deren äusseres bis zum Geschwulsthals zu verfolgen ist, wo man nicht selten feste Verlötungen mit der Glaslamelle antrifft.

Das innere Blatt, welches mit der Hauptmasse der Retina den Rücken des Sarkomknotens überzieht, ist nur auf kurze Strecke in stark rarefieirtem Zustand sichtbar. Besser erhalten sind die übrigen Netzhautschichten, namentlich die innere Körnersehicht, kenntlich an der reihenweisen Anordnung der Rundzellen. Nur der Scheitel des Geschwulstkopfes ist gänzlich von Netzlaaut entblösst und die Tumorzellen haben hier Gelegenheit, frei in den Glaskörper hinein $\mathrm{zu}$ wuchern. 
Die geschilderten Verhältnisse sind am besten auf der dem Sehnerveneintritt zugekehrten Seite des Sarkoms zu beobachten, während die von der vorderen Fläche absteigende Retina sehr bald in die Pars ciliaris retinae ubergeht, worunter die Klarheit des anatomischen Befundes leidet.

Erwähnen möchte ich noch, weil bei dem verhältnissmässig jugendlichen Alter der Patientin nicht gerade häufig, dass in den der Ora serrata zunächst gelegenen Retinaabschnitten sich die bekannte, von Iwan off als senile Veränderung beschriebene cystoide Degeneration in sehr ausgesprochenem Grade vorfindet.

Hinsichtlich der Aderhant ist auch in diesem Falle die mächtige Erweiterung des Venennetzes in der Umgebung des Tumors zu erwähnen.

Die Glaslamelle, welche an der Perforationsstelle ahnliche, wenn auch weniger deutliche Einrollungen wie in Fall 1 zeigt, weist in der Nachbarschaft der Neubildung sehr zahlreiche drusige Verdickungen mit starker Wucherung des Pigmentepithels anf.

Die der Gesehwulstbasis anliegenden Schichten der Sklera sind von Spindelzellen infiltrirt. Dieselben haben sich in regelmässigen Reihen in den Gewebsspalten der Sklera angeordnet, stellenweise in solcher Mächtigkeit, dass das Grundgewebe nur noch als spärliche feine Fasern sich zu erkennen giebt. Das Fortwandern von Tumorzellen auf dem Wege von Gefässscheiden konnte hier nicht beobachtet werden.

Die übrigen Angentheile, speciell auch Sehnerv und Kammerwinkel, verhalten sich durchaus normal.

Die im Vorstehenden beschriebenen Fälle weichen in verschiedener Beziehung von dem gewöhnlichen Bilde der Aderhautsarkome ab. Wenn ich zunächst das klinische Verhalten kurz streife, so gehören beide nach der zuerst von $\mathbf{K}_{n a p p^{1}}$ ) präcisirten Eintheilung dem ersten durch reizlosen Verlauf und Beschränkung auf das Bulbusinnere charakterisirten Stadium an. Wie nicht anders zu erwarten ist, sind anatomische Untersuchungen in diesem. Frühstadium ziemlich selten. So sind unter der 196 Fälle umfassenden Fuchs'schen ${ }^{2}$ ) Zusammenstellung nur zwölf hierher gehörig. In neuerer Zeit sind freilich, Dank den verfeinerten Untersuchungsmethoden, solche Frühdiagnosen häufiger, bilden aber trotzdem noch einen recht geringen Procentsatz der Gesammtzah].

Namentlich mein erster Fall befindet sich noch auf einer ungewöhnlich frühen Entwicklungsstufe und dadurch zum Studium der anatomischen Verhältnisse ganz besonders geeignet.

Ich will nun auf letztere in einigen Punkten näher eingehen.

Die mikroskopische Untersuchung hat in Fall 1 ein fascicu. läres Spindelzellensarkom ergeben, welches hinsichtlich des Pig-

1) H. Knapp, Die intraocularen Geschwülste. 1868.

2) E. Fuchs, Das Sarkom des Uvealtractus. 1882. 
mentgehaltes Zweifel erregen könnte, ob man es mit einem Melanosarkom zu thun habe. Wenn auch eine pathologische Vermehrung des normalen präformirten Stromapigmentes zugegeben werden muss, so ist sie meines Erachtens durch eine reactive Wucherung des pigmenthaltigen Stromagewebes hinlänglich erklärt und berechtigt nicht, die Geschwulst deshalb als melanotisch zu bezeichnen.

Der zweite Fall ist ein wahres Leukosarkom mit den besonderen Eigenschaften des Angiosarkoms. Letztere Geschwulstform wurde im Auge zuerst im Jahre 1868 gleichzeitig von Leber ${ }^{1}$ ) und $\mathrm{Knapp}{ }^{2}$ ) nachgewiesen, später aber als ein so überaus bäufiges Vorkommniss erkannt, dass man heute ein mehr oder weniger deutliches Hervortreten des angiomatösen Typus geradezu als die Regel ansieht. - Das histologische Bild des vorliegenden Falles wird noch wesentlich complicirt durch das oben beschriebene System von Spalträumen, welches den Tumor durchsetzt.

Die Beziehungen, welche dieselben zu den Blutgefässen erkennen lassen, sowie ihr Inhalt machen es sehr wahrscheinlich, dass es sich hier um eine ausgedehnte Proliferation der die Blutgefässe umgebenden Lymphräume handelt.

Näher möchte ich weiterhin auf das höchst eigenthümliche Verhalten der Netzhaut eingehen, welches in beiden Fällen, am ausgesprochensten bei Fall $1 \mathrm{zu}$ beobachten ist. Ich meine die Spaltung der äusseren bezw. der inneren Körnerschicht in zwei Lamellen, die den extrachorioidealen Geschwulstknoten kapselartig umhüllen, und deren äussere an der Durchbruchsstelle der Lamina elastica deutliche Verwachsungen mit dieser aufweist. Ich glaube diesen Befund in folgender Weise erklären zu können. Da eine active Thätigkeit der Retina, ein Umwachsen der Neubildung von vorn herein auszuschliessen ist, so kann es sich nur um ein Hineinwuchern der Geschwulst zwischen die Retinaschichten handeln. In einem sehr frühen Stadium, vor Sprengung der Glaslamelle und vor der Abscheidung eines subretinalen Exsudates hat der sarkomatöse Process vermuthlich zu einer entzündlichen Verwachsung zwischen der bedeckenden Aderhaut und den nächstliegenden Schichten der Retina geführt. Wie diese Fernwirkung zu Stande kommt, ob dabei chemotactische Einflüsse der Stoffwechselproducte des Tumors eine Rolle

1) Th. Leber, Fall von cavernösem Sarkom der Aderhaut. v. Graefe's Arch. f. Ophthalm. Bd. XIV. 2.

2) H. Knapp, loc. cit. Fall 14. S. 134.

v. Graefe's Archiv für Ophthalmologie. LIV. 3. 
spielen, will ich hier nicht weiter erörtern; es genügt, dass durch zahlreiche Beobachtungen das Vorkommen solcher Verwachsungen gesichert ist. Dem Andrängen der wuchernden Geschwulstmasse musste die Glaslamelle schliesslich nachgeben und platzte. Gleichzeitig mit ihr zerriss die Retina, so weit sie mit ihr unlöslich verlöthet war, nämlich die Pigmentschicht und - ich muss dies supponiren - die äusseren Zelllagen der äusseren Körnerschicht (Textfigur 2). Der Tumor befand sich nun in unmittelbarem Contact mit der Retina und zwar mit dẹm - nicht zerrissenen - Rest der äusseren Körnerschicht. Verwachsungen dieser beiden Gewebe unter einander waren auf die Dauer unausbleiblich. Dem unaufhaltsamen weiteren Wachsthum der Geschwulst musste die Retina nachgeben. Während also die inneren Netzhautschichten sich immer mehr von der Aderhaut entfernten, wurden Pigmentepithel und die nächsten

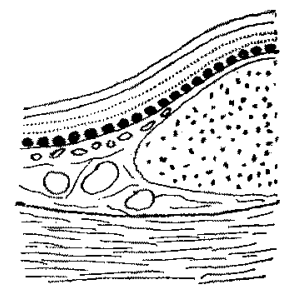

Fig. 1.

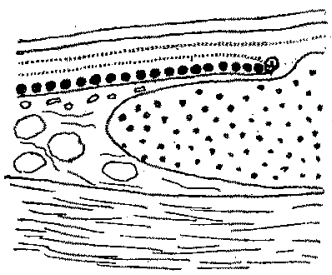

Fig. 2.

Lagen der äusseren Körner durch ibre unnachgiebigen Verwachsungen an der Perforationsstelle festgehalten. Dies musste zu einer förmlichen Spaltung der äusseren Körnerschicht in zwei Blätter führen, zwischen die sich der Tumor hineindrängte. Unterdessen hatte sich im nächsten Umkreis ein bei Aderhautsarkomen selten ganz fehlendes subretinales Exsudat angesammelt und zu einer umschriebenen Abhebung der Netzhaut mit Ausnahme des Pigmentepithels geführt, welch letzteres, wie stets, an der Aderhaut haften geblieben war (Textfig. 3). Aber auch diese neue Zugkraft war nicht im Stande, jene Verlöthungen zu zerreissen, die in meinen beiden Fällen ganz ungewöhnlich fest gewesen sein müssen. Wohl aber mussten sich die betroffenen Netzhautschichten hochgradige Zerrungen gefallen lassen. Thre Zellen verloren ihren Rundzellencharakter und wurden zu langen dünnen, Bindegewebsfasern gleichenden Zellen ausgezogen. Von dem zunehmenden subretinalen Exsudat gegen die Seitenflächen des Tumors gepresst (Textfig. 4), verklebten sie mit diesem mehr oder weniger fest, auf diese Weise eine Umhüllungsmembran bildend. Die etwas 
abweichenden Verhältnisse am oberen Tumorrande, wo die innere Körnerschicht die analogen Veränderungen aufweist, stehen dieser Erklärung nicht im Wege. Hier waren die anfänglichen Verwachsungen tiefer greifend und erstreckten sich noch auf einen Theil der inneren Körnerschicht. Dieser, sowie die gesammte äussere Körnerschicht zerriss gemeinsam mit der Glaslamelle, der Rest der inneren Körner und die übrige Netzhaut wurde vom Tumor abgehoben.

Dass ich fast gleichzeitig in zwei Fällen dieser doch immerhin nicht häufigen Augenaffection ein so uibereinstimmendes Verhalten der Netzhaut constatiren konnte, legte die Vermuthung nahe, dass es eine nicht besonders seltene Erscheinung sei. Ich suchte nun in der mir zugänglichen Sarkomliteratur - auf nicht weniger als 200

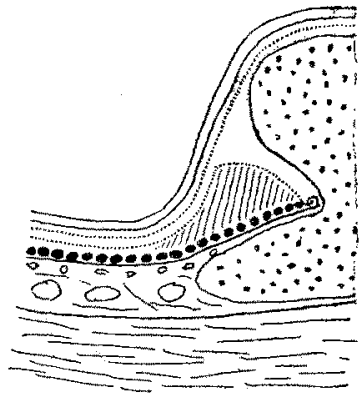

Fig. 3 .

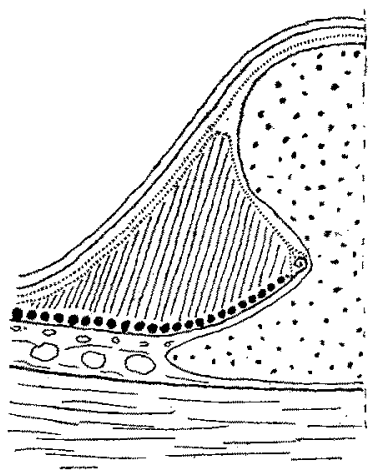

Fig. 4.

Arbeiten haben sich meine Nachforschungen erstreckt - nach entsprechenden Befunden, aber mit durchaus negativem Erfolg, so dass ich meine beiden Beobachtungen wohl mit gutem Grund als Seltenheiten bezeichnen kann.

Bekanntlich gilt als eines der häufigsten Frühsymptome der Aderhautsarkome eine totale, trichterförmige Netzhautablösung, von Arlt treffend mit der Form einer Windenblüthe verglichen. Noch im Jahre 1866 erklärte A. v. Graefe ${ }^{3}$ dies für die Regel. Jedoch bemerkt schon Knapp ${ }^{2}$ ): „Die Netzhaut überkleidet den Tumor von Anfang an und verwächst gewöhnlich locker mit ihm, während sie in ihrem übrigen Abschnitt meistens schon frühzeitig abgelöst wird." Im gleichen Jahre, gelegentlich des Heidelberger Ophthalmologen-

1) A. v. Graefe, Vereinzeltes über Tumoren. v. Graefe's Arch. f. Ophthalm. Bd. XII. 2. S. 238.

2) Die intraocularen GeschwüIste. 1868 . 
congresses wies derselbe Autor ${ }^{1}$ ) darauf hin, dass Sarkome des hinteren Bulbusabschnittes, speciell der Maculagegend, durchaus nicht so selten ohne Netzhautablösung verlaufen, und hielt seine Behauptung trotz des Widerspruches de Wecker's aufrecht. Endlich brachte dasselbe Jahr (1868) einen von Hirschberg ${ }^{2}$ ) publicirten Fall, in dem bei macularem Sitz der Geschwulst die Netzhaut fest anlag. Eine kräftige Stiitze erhielt die Knapp'sche Anschauung zwei Jahre später durch Becker ${ }^{3}$ ), der in der Lage war, sieben Fälle, darunter drei mit macularem Geschwulstsitz, mitzutheilen, in denen sämmtlich eine Netzhautablösung fehlte. Nachdem einmal die Aufmerksamkeit auf diesen Punkt gelenkt war, wurden von nun an derartige Fälle häufiger veröffentlicht, ich weise namentlich auf eine Anzahl von Knies ${ }^{4}$ beobachtete hin, in denen die Abhebung ganz fehlte oder, wie in den meinigen, sich nur auf die nächste Umgebung des Neoplasmas beschränkte.

Die Netzhaut war dabei bald der Geschwulst lose aufliegend, bald mit ihr mehr oder weniger innig verwachsen. Nirgends jedoch fand ich eine Notiz, die auf eine Spaltung der Retinaschichten schliessen liess.

Wie es scheint vereinzelt steht eine Beobachtung ron $\mathrm{Knapp}{ }^{5}$ ): Eine knopfförmige Geschwulst im hinteren Bulbusabschnitt, von welcher die der Aderhaut überall anliegende Retina perforirt war. Aehnlich wie bei meinen Fällen muss man auch hier eine sehr frühzeitige Verlöthung der Aderhaut mit der Retina annehmen. Nur muss hier der Zusammenhang der Retinaschichten unter einander ein so fester gewesen sein, dass bei dem später erfolgenden Durchbruch der Glaslamelle die Retina in ihrer ganzen Dicke mit derselben gleichzeitig zerriss.

Besondere Beachtung verdient das histologische Verhalten des Pigmentepithels. Längst bekannt und von vielen Autoren hervorgehoben ist die Betheiligung des auch den Tumor nicht unmittelbar begrenzenden Pigmentepithels an dem Krankheitsprocess, die sich in

1) Knapp, Ueber Tumoren. Sitzungsbericht der ophthalm. Gesellsch. 1868.

2) J. Hirschberg, Casuistische Mittheilungen über Geschwülste der Orbita und des Bulbus. Zehender's Monatsbläter. Bd. VI.

3) O. Becker, Zur Diagnose intraocularer Sarkome. Arch. f. Augenheilk. Bd. I. 2.

4) M. Knies, Sechzehn Fälle von Aderhautsarkom nebst epikritischen Bemerkungen. Arch. f. Augenheilk. Bd. VI.

5) Intraoculare Geschwülste. 1868. Fall 14 . 
der verschiedensten Weise äussern kann. Bald sind es blosse Unregelmässigkeiten in der Grösse der einzelnen Epithelzellen, bald findet man diffuse oder umschriebene Wucherungen bis zur Entstehung kugeliger, aus Pigmentzellen bestehender Excrescenzen, bald sind einzelne Zellen ganz aus dem Verbande gelöst und schwimmen frei in dem subretinalen Exsudat umher. Derartigen Bildern begegnet man in meinen beiden Fällen sehr häufig. Es scheint, dass das Auftreten dieser Störungen des Pigmentepithels an das Vorhandensein eines Exsudates gebunden ist, wenigstens waren sie stets auf den Bereich der pathologischen Netzhautablösung beschränkt und börten an ihrer Grenze ganz unvermittelt auf, so dass die Bespülung mit dieser eiweissreichen Flüssigkeit als formativer Reiz auf das Pigmentepithel gewirkt haben dürfte.

Ferner machte Fuchs ${ }^{1}$ ) auf das häufige Vorkommen von drusenartigen Veränderungen der Glashaut mit den entsprechenden Veränderungen des Pigmentepithels aufmerksam. Auch hierfür bieten meine beiden Fälle zahlreiche Beispiele.

Weit interessanter aber, und nicht so einfach zu erklären ist eine dritte Art von Knötchenbildung des Pigmentepithels, die Fall 1 in mässiger Anzahl aufweist. Ich meine die umschriebenen tumorartigen Anhäufungen nicht pigmentirter Zellen zwischen Glaslamelle und dem unveränderten Pigmentepithel gelegen, und von letzterem überzogen oder dasselbe an der Spitze durchbrechend. Eine gleichartige Beobachtung wurde bereits im Jahre 1874 von $\mathrm{Knapp}{ }^{2}$ ) veröffentlicht und abgebildet:

Bulbus einer 40jährigen Fran, enucleirt zwei Jahre nach Beginn der ersten Krankheitserseheinnngen. Pigmentirtes Rundzellensarkom im hinteren Abschnitt mit grossem secund̈rem Orbitaltumor. Netzhaut total abgelöst und mit zahlreichen Sarkomknötchen durchsetzt. Innenfläche der Aderhaut zu einem Viertheil ihrer ganzen Ausdehnung von kleinen bis hirsekorngrossen, runden flachen oder halbkugeligen Knötchen dicht besät. Mikroskopisch bestanden die Knötehen aus derselben Art von Zellen, wie der ursprüngliche Tumor, waren also als Dissemination des Sarkoms aufzufassen.

Die Uebereinstimmung der Knapp'schen Zeichnungen mit den meinigen ist augenfällig, wenn man berücksichtigt, dass es sich bei Knapp um die Wiedergabe ungefärbter, bei mir um gefärbte Präparate handelt. Die Wesensgleichheit unserer Befunde kann danach

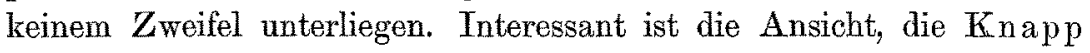

I) Das Sarkom des Uvealtractus. 1882. S. 216.

2) E. Williams und H. Knapp, Zwei eigenthümliche Falle von Chorioidealsarkom. Arch. f. Augenheilk. Bd. IV. S. 83. 
über die Entstehung dieser Bildungen äussert: „Von den Knötchen in der Retina lösten sich Zellen ab und fielen in die zwischen Retina und Chorioidea befindliche Flüssigkeit, um von da auf die Epithelschicht der Chorioidea abgelagert zu werden. Hier schlugen sie Wurzel, und da sie in ihrer Ernährung von der Choriocapillaris abhängig waren, so ist es nicht auffallend, dass sie die Epithelschicht durchdrangen, aber durch die zwischen Epithelschicht und Choriocapillaris befindliche byaline Membran von weiterem Vordringen abgehalten wurden. Während sie sich nun vermehrten, verbreiteten sie sich zwischen den beiden genannten Schichten, hoben die Epithelschicht empor, durchbrachen sie und wucherten in den Glaskörperraum."

Der Knapp'sche Fall hat als Beispiel für eine Bildung secundärer Geschwülste durch Dissemination von Geschwulstkeimen eino gewisse Rolle in der Literatur der letzten Jahre gespielt und alle sich mit diesem Gegenstand beschäftigenden Arbeiten greifen auf dieselbe zurück. Als erster befasste sich Mitvalsky ${ }^{1}$ ) 1894 wieder mit dieser Frage.

Es handelte sich um ein flächenhaftes Sarkom der Maculagegend, welches auf die benachbarte Retina übergegriffen und in den vorderen Netzhautpartien zu mehreren Secundärknoten geführt hatte, deren "Gebundensein an das Pigmentepithel überall mehr als ersichtlich war". Durch Serienschnitte liess sich nachweisen, „dass das zwischen dem primären Sarkomknoten und den secundären prääquatoriellen Disseminationsknötchen intercalirte Pigmentepithel in toto, auf seiner ganzen Strecke vermehrt, meistens vervielfältigt, geschichtet und verschiedenartig verzweigt erscheint". Es kamen also „diese Disseminationsknötchen durch directe Fortsetzung der Zellen des primären Sarkomknotens in dem Pigmentepithel nach vorne und durch ihre Vermehrung daselbst zu Stande".

Auf Grund dieses Befundes zieht Mitvalsky die von Knapp gegebene Erklärung in Zweifel und glaubt, auch den Knapp'schen Fall auf directes Fortwuchern der Sarkomzellen innerhalb des Pigmentepithels zurückführen zu müssen.

Weiterhin beschäftigte sich $\mathrm{Ewetzky}^{2}$ ) mit diesem Gegenstand, der in einem Fall von Melanosarcoma iridis mit Ausbreitung auf den Ciliarkörper und die Chorioidea zahlreiche pigmentirte, lebensfähige Geschwulstzellen im Glaskörper, besonders im vorderen Theil

1) Zur Kenntniss der Aderhautgeschwülste. Arch. f. Augenheilk. Bd. XXVIII.

2) Th. Ewetzky, Ueber Dissemination der Sarkome des Uvealtractus. v. Graefe's Arch. f. Ophthalm. Bd. XLII. 1. 
antraf, „bei denen jeglicher Gedanke fern liegen musste, sie als Derivate des retinalen Pigmentepithels zu betrachten". Durch Ablagerung und Proliferation solcher Zellen soll in der Netzhaut an einer Stelle ein kleines secundäres Sarkomknötchen entstanden sein.

Erwähnen möchte ich noch die Arbeiten von Vose Solomon ${ }^{1}$ ), Webster und J. van Gieson ${ }^{9}$, Robertson und Knapp ${ }^{3}$, Kamocki ${ }^{4}$ ), Gerken ${ }^{5}$ ), die sämmtlich Fälle von Ciliarkörper- oder Irissarkomen mit Dissemination in den nächstliegenden Theilen, Kammerwinkel, vordere Linsenkapsel etc, behandeln.

Meine Beobachtung scheint mir geeignet zu sein, zur Klärung dieser noch strittigen Fragen beizutragen. Aus verschiedenen Gründen glaube ich jene Zellanhäufungen unter dem Pigmentepithel unbedenklich als disseminirte Geschwulstknötchen ansprechen zu können. Mit einfachen Pigmentepithelwucherungen, wie sie gerade in meinen Präparaten sehr häufig sind, sind sie in keiner Weise zu verwechseln. Während diese ganz unregelmässig gestaltete lockere Anhäufungen durchweg pigmentirter Zellen oft von bedeutender flächenhafter Ausdehnung darstellen, sind jene Sarkomknötchen völlig scharf "umgrenzte kugelige Gebilde, frei von jedem Pigment. Ihre Kerne unterscheiden sich in nichts von denen junger Sarkomzellen, bei welchen die spindelförmige Gestalt noch nicht zur völligen Ausbildung gelangt ist, während sie in Gestalt und Grösse von den Kernen der Pigmentepithelzellen nicht unerheblich differiren. Auch Knapp war ja über die sarkomatöse Natur seiner gleichartigen Gebilde keinen Augenblick in $Z$ weifel.

Weit mehr Schwierigkeiten macht die Genese dieser Metastasen.

Die erste Möglichkeit: Auswanderung von Geschwulstzellen aus dem Aderhautstroma durch die Glaslamelle hindurch und Proliferation zwischen dieser und dem Pigmentepithel, ist ganz von der Hand zu weisen. Zunächst wurde die Glaslamelle stets intact gefunden; dann wäre es unverständlich, wie die homogene feste Membran, die dem wachsenden Tumor so lange Widerstand zu leisten vermochte, ohne ersichtlichen Grund von Geschwulstzellen durchwandert werden sollte. Endlich finden sich die Metastasen nicht in der Nähe des

x) Melano-sarcomatous tumour growing from the iris of the right eye. Transact. of the Ophthalm. Society of the Unit. Kingd. Vol. II. 1882.

$\left.{ }^{2}\right)$ A case of sarcom of the iris. Arch. of ophthalm. Vol. XVIII.

$\left.{ }^{3}\right)$ Sarkom der Iris. Areh. f. Augenheilk. Bd. III. 2.

4) Ein Fall von disseminirtem Uvealsarkom. Zeitschr. f. Augenheilk. Bd. III.

5) Gerken, Beitrag zur Kenntniss des primären Irissarkoms. Dissert. 1900. 
Tumors, wo die Aderhaut von Sarkomzellen infiltrirt ist, sondern gerade in weiterer Entfernung von demselben, an Stellen, wo in weitem Umkreis im Aderhautstroma keine Geschwulstzellen mehr anzutreffen sind.

Ebenso wenig kann ein directes Fortwuchern der Sarkomzellen innerhalb des Pigmentepithels, wie es Mitvalsky beschreibt, in Betracht kommen, denn auf der ganzen zwischen den Disseminationsherden und dem Primärtumor liegenden Strecke zeigt das Pigmentepithel, wie die Serienschnitte unwiderleglich beweisen, keinerlei Andeutung einer Infiltration mit Sarkomzellen. Bei der grossen Aehnlichkeit zwischen den von Knapp beschriebenen Metastasen und den meinigen, muss ich diese Art der Entstehung auch für jene ausschliessen und kann darin Mitvalsky nicht beistimmen, der den für seinen Fall zutreffenden Entwicklungsmodus auch für Knapp's Fall postuliren will.

Wir stehen also vor der Wahl, entweder die von Knapp gegebene Erklärung - Einwanderung von Geschwulstzellen vom subretinalen Exsudat aus in das Pigmentepithel - als richtig anzuerkennen, oder eine selbständige sarkomatöse Degeneration des Pigmentepithels anzunehmen.

Die letztere Hypothese würde zwar mit der durch Leber's Untersuchungen ${ }^{1}$ ) wahrscheinlich gemachten Betheiligung des Pigmentepithels an dem Aufbau des Sarkoms in Einklang stehen, wird jedoch durch den anatomischen Befund nicht genügend gestïtzt. Das den Ueberzug der Knötchen bildende Pigmentepithel lässt in keiner Weise eine active Mitwirkung an ihrer Entstehung erkennen; auch spricht der Umstand, dass sie vorzugsweise in grösserer Entfernung vom Haupttumor anzutreffen sind, gegen eine derartige Histogenese ${ }^{2}$ ).

Wohl aber sind alle Vorbedingungen erfuillt, um sie auf ver-

1) Th. Leber, Veber die Aderhautsarkome und die Herkunit ihres Pigmentes. v. Graefe's Arch. f. Ophthalm. Bd. XLIV. 3.

2) Seit der Veröffentlichung meiner oben erwähnten Beobachtungen habe ich in Fällen von Aderhautsarkom, die wie die eben beschriebenen frühzeitig zur Enucleation kamen, mich regelmässig überzeugen können, dass von einer activen Betheiligung des Pigmentepithels an dem Wucherungsprocess im ersten Stadium nicht die Rede sein kann. Ich möchte hiernach annehmen, dass die von mir beschriebenen Vorgänge von. Seiten des Pigmentepithels als secundär zu betrachten sind. Mit den in der vorliegenden Arbeit beschriebenen Knötchenbildungen haben aber meine Beobachtungen nichts gemein; die Auffassung der ersteren als durch Dissemination entstandener miliarer Sarkommetastasen halte ich für vollkommen berechtigt.

Th. Leber. 
sprengte Geschwulstkeime zurückzuführen. Wie ich schon oben hervorhob, steht das Sarkom an einigen Stellen in unmittelbarem Contact mit der subretinalen Flüssigkeit und zeigt gerade hier in Folge des an dieser Stelle unbehinderten Wachsthums besonders lockeres Gefüge. Die Möglichkeit, dass sich hier Geschwulstzellen ablösten und durch das flüssige Exsudat nach entfernten Stellen transportirt wurden, muss ohne Weiteres zugegeben werden. Es gelang auch unschwer, in der Exsudatfliüssigkeit solche Zellen nachzuweisen und zwar am zahlreichsten nahe ihrem oberen Rande, wo man sie in ziemlicher Anzahl theils einzeln, theils in Verbänden von drei oder vier Zellen antrifft. Sie haben sich hier der äusseren Fläche der abgehobenen Retina angelagert, ohne in dieselbe einzudringen. $\mathrm{Zu}$ beachten ist nun, dass von den sieben Disseminationsherden, die ich nachweisen konnte, nicht weniger als sechs sich in unmittelbarer Nähe dieser versprengten Zellen, also nahe dem oberen Rande der Netzhautablösung befanden, während nur eins sich in grösserer Entfernung von demselben vorfand. In welcher Weise sich das Eindringen der Geschwulstzellen in das Pigmentepithel vollzogen hat, welche Momente hierbei als treibend anzusehen sind, darüber gewährten meine Präparate keinen Aufschluss. Es steht aber nichts im Wege, nach dem Vorgang von Knapp eine active Thätigkeit der Zellen mit dem Zweck, günstigere Ernährungsbedingungen zu erlangen, anzunehmen.

Meinem hochverehrten Chef, Herrn Prof. Leber, erlaube ich mir, für das dieser Arbeit entgegengebrachte Interesse meinen aufrichtigsten Dank auszusprechen.

\section{Erklärung der Textfiguren 1-4, sowie der Abbildungen auf Taf. XIII, Fig. 1-3.}

Textfiguren 1-4 erläutern schematisch das Zustandekommen der Spaltung der äusseren Körnerschicht. Dieselbe ist als aus zwei Schichten bestehend gedacht und durch $z$ w ei punktirte Linien dargestellt. Von den übrigen Netzhautschichten sind die innere Körnerschicht und die Ganglienzellenschicht durch je eine schwarze Linie angedeutet.

Taf. XIII, Fig. 1. Mikrophotographische Aufnahme eines mit Hämatoxylin-Eosin gefärbten Präparates. Zeiss Apochrom. 16. Dargestellt ist die Berührungsstelle der abgelösten Netzhaut mit dem unteren Tumorrand (Fall I).

Fig. 2. Disseminirtes Sarkomknötchen. (Fall I) Färbung mit Hämatoxylin-Eosin. Leitz. Object. 7 . Ocul. 1.

Fig. 3. Desgl. Leitz. Oelimmersion 1/12. Ocul. 1. 


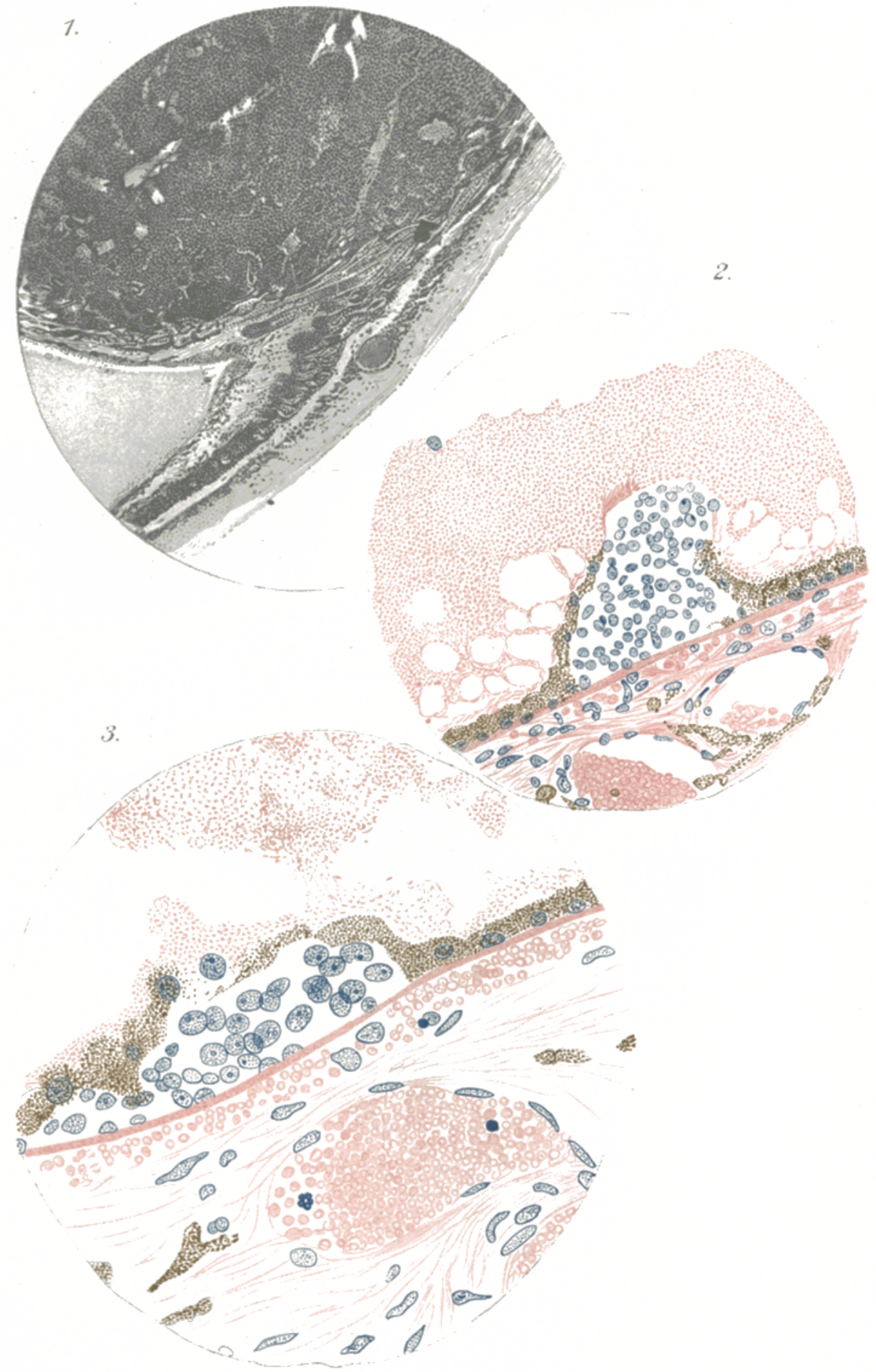

Brums delin 